\title{
Effect of bioactive coating of the tibial component on migration pattern in uncemented total knee arthroplasty: a randomized RSA study of 14 knees presented according to new RSA-guidelines
}

\author{
M. Therbo $\cdot$ B. Lund $\cdot$ K.-E. Jensen • \\ H. M. Schrøder
}

Received: 9 October 2007/ Accepted: 25 January 2008/Published online: 10 May 2008

(C) Springer-Verlag 2008

\begin{abstract}
Background Bioactive coating of uncemented total knee arthroplasty (TKA) is believed to increase bone ingrowth and enhance early fixation of the TKA. In a prospective randomized study using radiostereometric analysis (RSA) we examined migrations of the tibial implant, in an uncemented TKA with and without bioactive coating. The study was performed according to new RSA guidelines, and focus was put on some important methodological issues.

Materials and methods Twenty-three patients with osteoarthrosis of the knee received an uncemented Duracon TKA either with bioactive (hydroxyapatite or periapatite) coating (+HA) or without bioactive coating (-HA). Patients had RSA examinations postoperatively and at 3,6 and 12 months. Nine patients were excluded during the study resulting in 14 knees for final analysis.

Results At 12 months follow-up we found no significant differences in migrations between the two groups. However, in general the -HA group migrated more than the $+\mathrm{HA}$ group, and we found a significant larger variation in migration pattern in the - HA group. In the + HA group the tibia component stabilized after 6 months, whereas the -HA group showed continuous migration. Subsidence and posterior tilt were the main migration patterns in both groups.
\end{abstract}

M. Therbo $(\bowtie) \cdot$ B. Lund $\cdot$ H. M. Schrøder

Department of Orthopaedic Surgery U, Rigshospitalet,

National University Hospital, Blegdamsvej 9,

2100 Copenhagen, Denmark

e-mail: m.therbo@tdcspace.dk

K.-E. Jensen

Department of Orthopaedic Radiology X, Rigshospitalet, National University Hospital, Blegdamsvej 9,

2100 Copenhagen, Denmark
Conclusions Bioactive coating of TKA seems to enhance early stabilization of the tibia component. Similar results are found in previous studies.

Keywords Hydroxyapatite - Migration .

Roentgenstereogrammetric analysis .

Total knee arthroplasty · Tibial implant

\section{Introduction}

Early fixation of the tibial component after total knee arthroplasty (TKA) is crucial for long-term survival of the implant $[1,2]$. In uncemented TKA the bone ingrowth into the porous coated surface of the implant is inhibited by a motion-induced fibrous membrane between the bone and the implant surface. Bioactive coating of the implant surface with hydroxyapatite (HA) converts this fibrous membrane to bony anchorage across the surface gaps of the implant [3-6]. It has been a challenge to science to predict the long-term outcome of TKA. DEXA-studies of bone mineral density (BMD) adjacent to the TKA-implant is a valuable tool in detecting stress-shielding and the attendant risk of fractures, but do not seem to be a convincing tool in considering long-term stability of implants [7]. A study on differences in BMD between HA-coated and non-HA coated tibial implants have also been carried out and no significant differences were found [8]. Conventional X-ray examinations after TKA are a valuable tool in estimating two-dimensional orientation of the implant. Furthermore $\mathrm{X}$-ray examinations sometimes can be a supportive option when considering aseptic loosening (clearing up zones) of the implant. However, X-ray examinations used as a predictive tool for estimating long-term survival due to fixation of the implant are also of limited value. In 1974, Selvik developed Roentgensterogrammetric Analysis 
(RSA), a method for quantifying the fixation of an implant with high precision and accuracy by estimating three-dimensional (3D) movements (migrations) over time [9-11]. Since then more than 300 scientific papers dealing with the subject RSA have been published. Several studies have found RSA as a valuable method of predicting longterm aseptic loosening of implants after TKA [1, 2, 9-11]. However, some major problems due to differences in the technical procedures, terminology and presentation of data among several RSA-studies have been pointed out [12]. These differences between studies make it sometimes difficult to compare data from one study to another. To overcome these problems in the future six international research centers recently agreed upon new standards for terminology, description and use of RSA arrangement. The new standards are preliminary presented as "guidelines" by Valstar et al. in 2005 [12] and will form the basis of a later standardization protocol. Our study is widely based on these new guidelines and, when relevant, items in standardization of RSA of special importance to the reader of this article will be focused on.

\section{Materials and methods}

Twenty-three patients with osteoarthrosis of the knee were enrolled in a prospective randomized design study. All patients received a porous coated posterior cruciate ligament (PCL)-retaining uncemented Duracon ${ }^{\mathrm{TM}}$ TKA (Howmedica ${ }^{\circledR}$, Rutherford, USA). One group (+HA) received a tibial implant coated with bioactive hydroxyapatite (HA) and the other group (-HA) had no bioactive coating. The bioactive-coated implants were delivered from the manufacturer who prepared the implant either by plasmaspraying technique $(n=2)$ or watery bath technique $(n=5)$. The two techniques are known as hydroxyapatite- and periapatite techniques. No clinical randomized studies known to the authors have demonstrated any significant differences in migration of implants between the two techniques used. Nine patients were excluded from the study (Table 1). Thus, 14 patients $(+\mathrm{HA}=7$ and $-\mathrm{HA}=7)$ remained in the study with 12 months follow-up. Demographic data and preoperative knee score in the two groups are presented in Table 2. Standard operation procedure was used on all patients. Perioperatively six to eight tantalum balls (Wennebergs Finmekaniska, Sweden) with a diameter of $0.8 \mathrm{~mm}$ were inserted in the tibia polyethylene and in the proximal tibia bone, respectively, for later RSA-examinations. Postoperatively all patients followed the standard rehabilitation program in our department. Functional knee score (HSS) was registered preoperatively and at 12 months follow-up.
Table 1 Patients excluded from the study and the cause of exclusion

\begin{tabular}{ll}
\hline Patient & Cause of exclusion from the study \\
\hline 1 & $\begin{array}{l}\text { Femoral fracture (patient was reoperated). } \\
2,3\end{array}$ \\
4 & $\begin{array}{l}\text { Too few tantalum bone markers in tibia. Knee } \\
\text { calibration cage in wrong position. }\end{array}$ \\
5 & $\begin{array}{l}\text { Too few tantalum markers in the tibia component. } \\
\text { Condition number and rigid body error too high } \\
\text { in both segments. }\end{array}$ \\
$6,7,8$ & $\begin{array}{l}\text { Postoperative RSA-images lost in X-ray archive. } \\
9\end{array}$ \\
Postoperative and 3-month RSA-images lost in \\
X-ray archive.
\end{tabular}

Table 2 Patient data (mean and range)

\begin{tabular}{lll}
\hline & +Coating & -Coating \\
\hline Gender $(\mathrm{f} / \mathrm{m})$ & $5 / 2$ & $3 / 4$ \\
Age & $67(56-82)$ & $75(65-85)$ \\
Body mass index (BMI) & $29(21-36)$ & $29(27-33)$ \\
Preop. knee score & $28(8-42)$ & $17(1-33)$ \\
\hline
\end{tabular}

RSA-examinations: RSA examination was performed postoperatively (within 1 week limit) and at 3, 6 and 12 months. Six patients had double RSA examinations for estimating precision of our RSA setup. The RSA examinations were performed at our Department of Orthopaedic Radiology. Two mobile X-ray tubes were available. The patient was placed in a supine position with the operated knee placed in a calibration Plexiglas cage (Cage 21, Tilly Medical Products, Sweden) and the two X-ray tubes in bi-planar position each at a distance of approximately $100 \mathrm{~cm}$ from the corresponding X-ray film (Fig. 1). The radiation intensity at each RSA examination was 50 kilovoltage $(\mathrm{kV})$ and 20 milliampere $\times$ second $(\mathrm{mAs})$, and estimations from previous studies [11] with an equivalent experimental setup reveal that the total effective radiation dose throughout the study is only approximately $1 \%$ of the yearly natural background radiation. At each RSA examination the patient was examined in the same standardized position with the operated knee aligned to the global coordinate system. In this way it is possible to detect any migrations along and around the three orthogonal axes $(x, y, z)$. All subsequent calculations at our workstation were performed respecting right-hand coordinate system which means that we changed signs for translations $(t)$ and rotations $(r)$ in left-hand extremities at the relevant axes $\left(x_{\mathrm{t}}\right.$ and $y_{\mathrm{r}}$ and $\left.z_{\mathrm{r}}\right)$. The bi-planar digital $\mathrm{X}$-ray examination was performed simultaneously yielding two X-ray images (one for each plane) that were stored in the central hospital archive (PACS system) as DICOM files. Using a special software application (DICOM GATEWAY) the image files were transferred to our workstation (DELL Inspiron 8100/ screen resolution: $1600 \times 1200 \mathrm{dpi}$ ). 


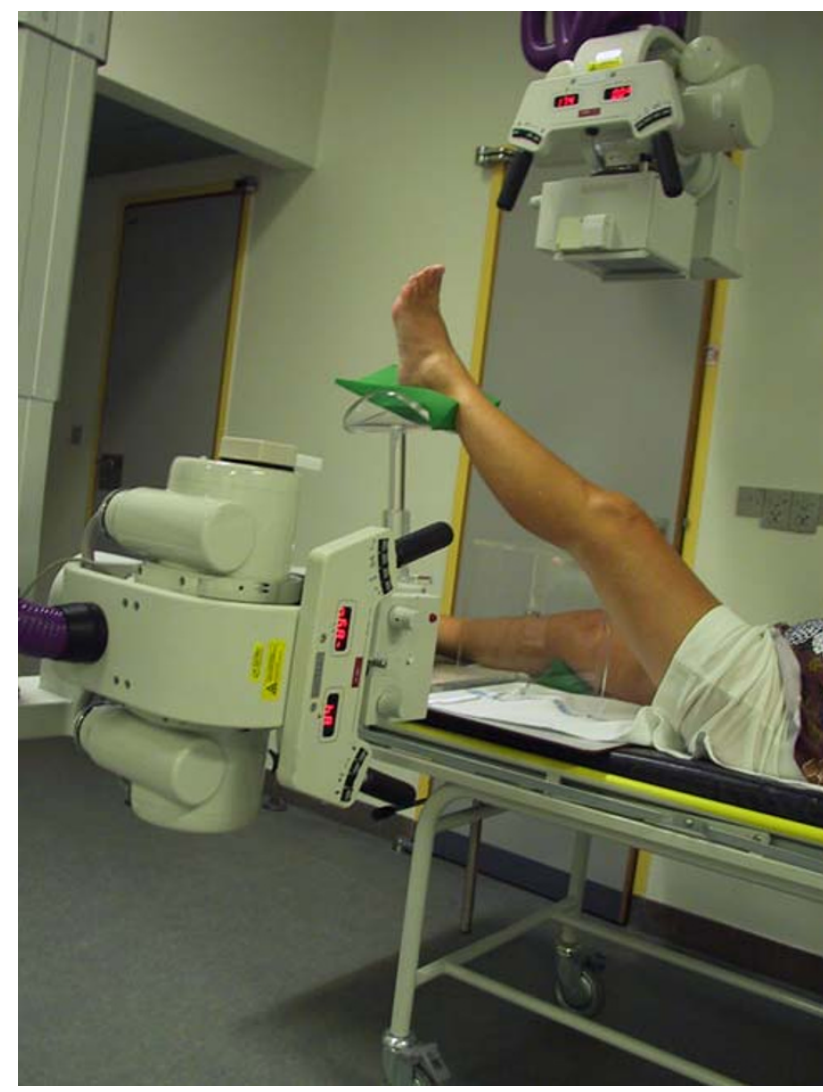

Fig. 1 Bi-planar RSA-setup with the two X-ray tubes at right angles and the patients right knee placed in the calibration knee cage. To gain overview scatter grids and X-ray film cassettes are not mounted

RSA-analyses: RSA-analyses were performed in our department at workstation using a validated [11] RSAsoftware program (WinRSA ver. 4.0, Tilly Medical Products, Sweden). The method for determining the position of the tibial implant in the global coordinate system arises from the kinematic model where the tantalum markers in the tibial implant and the proximal tibia bone define two rigid bodies (segments). The tantalum markers in the calibration knee cage define the global coordinate system. The proximal tibia bone acted as a reference segment for the tibial implant segment. The tantalum markers in both segments and the calibration knee cage were detected manually on the X-ray images in the two planes. It is of crucial importance that the corresponding marker is detected in the two planes and that a minimum of three corresponding markers are detectable in each segment. By mathematical transformation (interpolation of marker coordinates in the two planes) into the 3D laboratory coordinate system the RSA-software calculated the 3D position of the segments. Subsequently the migration of the tibial implant over time (according to follow-up schedule) was calculated with the postoperative examination as reference. Manual detection of markers is time consuming, and the mean time spent on one RSA examination and subsequent analysis of the RSA-image pair was $120 \mathrm{~min}$. The unit for translations was millimeters $(\mathrm{mm})$ and for rotations it was degrees $\left(^{\circ}\right)$. In our study the tibial implant was defined stable if the translation between two examinations were less than $0.2 \mathrm{~mm}$. In RSA examinations and analyses several factors influence the reliability of the results $[10,11,13]$. Two important parameters that affect the results of RSA analysis are the condition number and rigid body error.

Condition number $(C N)$ : When calculating the RSA results the RSA program also tests the distribution of tantalum markers in each segment and the mathematical expression for this spatial distribution is the CN [14]. A low $\mathrm{CN}$ indicates a wide spatial distribution of markers, whereas a high $\mathrm{CN}$ indicates a narrow (close to linear) distribution. A high $\mathrm{CN}$ affects the reliability of RSA results in a negative way. RSA guidelines [12] propose an upper limit (cut-off level) for $\mathrm{CN}$ of 150. If $\mathrm{CN}$ in an RSA examination exceeds this perceptible cut-off level this examination must be excluded from the study. In our study the chosen cut-off level for $\mathrm{CN}$ was 161 , which is very close to the recommended value. Moreover, in our study the mean CN values in all RSA analyses were 51 (95\% CL: 32-70) and 69 (95\% CL: 63-75) for the tibial implant- and proximal tibia segments, respectively, and in only one case (follow-up examination) the $\mathrm{CN}$ value was beyond 150 .

Rigid body error $(R B E)$ : From a kinematic point of view the segment is regarded as a rigid body. If for example one or more markers in a segment moves between two examinations there will be an RBE (deformity) in the segment that strongly affects the reliability of the kinematic analysis. Guidelines [12] propose a maximum mean rigid body error of $0.35 \mathrm{~mm}$. In our study the mean RBE was $0.10 \mathrm{~mm}$ (95\% CL: $0.05-0.16 \mathrm{~mm})$ and $0.11 \mathrm{~mm}(95 \%$ CL: $0.07-0.16 \mathrm{~mm}$ ) in the tibial implant and proximal tibia bone, respectively.

Statistics: Statistical software program SPSS version 14.0 was used. Differences in migrations and knee score between the two groups over time were evaluated by non-parametric test (Mann-Whitney $U$ test). To compare the variability in migrations between the two groups we performed a homogeneity test (Levene's test). $P$-values below 0.05 were considered significant. Prior to the study we did a sample size calculation with type- 2 error of $20 \%$ and MIREDIF $0.20 \mathrm{~mm}$. From previous studies we found SD from 0.10 to $0.20 \mathrm{~mm}$. Thus, a total of 30 patients were planned to be included in our study. However, due to delivery problems concerning some of the prostheses we were able to include only 23 patients. Nine patients were excluded from the study and because only seven patients in each group were left we performed no power analysis on these. A previous randomized study [15] very similar to our study with 26 patients 
showed a statistical power of $68 \%$. Our study has been approved by the local Ethical Commitee of Copenhagen and Frederiksberg, and informed consent was obtained from the patients prior to inclusion in the study.

\section{Results}

At 12 months follow-up we found no significant differences in mean translations and rotations between the two groups (Fig. 2). In the +HA and - HA group the tibial implant had subsided $-0.22 \mathrm{~mm}$ (range: -0.95 to 0.19 ) and $-0.49 \mathrm{~mm}$ (range: -2.57 to 0.87 ) after 12 months. In the -HA group we found a significantly larger translation along the $z$-axis at 3 months follow-up, and at 6 months we found a significantly larger translation along the $z$-axis as well as the $y$-axis (Fig. 2). From 6 to 12 months follow-up we found mean total translations (all three cardinal axes) of more than $0.20 \mathrm{~mm}$ in the $-\mathrm{HA}$ group, whereas in the +HA group we found mean total translations less than $0.20 \mathrm{~mm}$. In both groups at 12 months follow-up we found rotations (posterior tilt) around the $x$-axis as the main rotation pattern. These rotations reached a mean of $-0.50^{\circ}$ (range: -1.47 to 0.03 ) and $-0.97^{\circ}$ (range: -4.04 to 0.35 ) in the $+\mathrm{HA}$ and $-\mathrm{HA}$ group, respectively. Rotations around the $y$ - and $z$-axis were small. We found a significantly larger variation (Levene's test) in translations along the $z$-axis $(P=0.037)$ and rotations around the $z$-axis $(P=0.038)$ in the - HA group. Furthermore the variations in mean migrations in general were found larger in the - HA group than in the + HA group. We did double

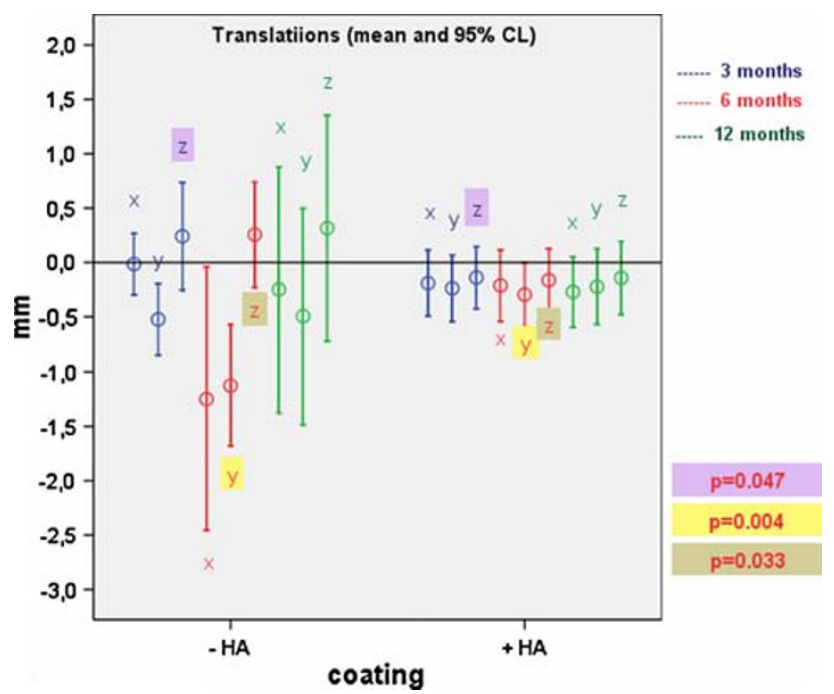

Fig. 2 Translations along the three cardinal axes after 3, 6 and 12 months for the two groups. $P$-values (Mann-Whitney $U$ test) indicates significant findings. To gain visual overview mean values are presented with $95 \% \mathrm{CL}$
RSA examinations of six knees and found a maximal $95 \%$ confidence limit (CL) reaching $0.08 \mathrm{~mm}$ and $-0.18^{\circ}$ for translations and rotations, respectively. Functional knee score at 12 months follow-up was 83 (71-96) and 81 (7587) for the +HA and -HA group, respectively, and showed no significant difference between the two groups.

\section{Discussion}

This study is the first in-house clinical RSA study from our department. Due to these facts we must conclude that several methodological and technical problems are to be solved when starting a new RSA study. We decided to refer widely to new guidelines for RSA. We find this study to be of great importance to any research group who consider commencing a new RSA study in the future. We find that our validated RSA system with high accuracy and precision is suitable for detecting 3D migrations of the tibial implant after TKA. In our study eight patients were excluded due to failure in the RSA procedure. However, in previous studies introducing in-house RSA techniques exclusion rates at a similar level were revealed $[1,13]$. The small number of patients in this study constitutes a risk of a considerable type- 2 error which means that our results must be looked upon with reservation. Furthermore we present a relative short follow-up period of only 1 year. Despite these facts we find a main migration pattern (subsidence and posterior tilt) of our tibial implants that are very similar to those migration patterns found in previous studies [13, 16-18]. In our study the tibial implant in the + HA group stabilized after 6 months, whereas in the -HA group the tibial implant showed continuing migration. Nelissen et al. [19] found in a randomized 2-year follow-up study of 30 TKA that the tibial implant in the HA-coated group migrated significantly less than that in the non-HAcoated group, and that the uncemented HA-coated implants migrated with similar magnitude as cemented tibial implants. Another randomized study [15] of 26 uncemented Duracon TKA with or without periapatite coating showed a clear tendency towards less migrations and variations in subsidence in the coated group compared with the non-coated group after 2 years. However, no significant differences in migrations between the two groups were found. In the mentioned study all patients suffered from rheumatoid arthritis which must be taken into account when comparing the results with our study where no patients suffered from rheumatoid arthritis. In the light of the results from our study we conclude that bioactive coating of uncemented TKA should be the standard. Further clinical studies, involving a greater number of patients and with longer follow-up period (a minimum of 2 years), of the differences in migrations between the bioactive 
coated and the non-coated TKA should be carried out. It is of great importance to evolve a standard protocol for RSA in the future to make it possible to compare different RSA studies. Until then the new RSA guidelines should be followed as widely as possible.

Conflict of interest statement The authors declare that they have no conflict of interest related to the publication of this manuscript.

\section{References}

1. Ryd L, Albrektsson BEJ, Carlsson L, Dansgård F, Herberts P, Lindstrand A, Regner L, Toksvig-Larsen S (1995) Roentgen stereophotogrammetric analysis as a predictor of mechanical loosening of knee prostheses. J Bone Joint Surg Br 77:377-383

2. Ryd L, Lindstrand A, Stenström A, Selvik G (1990) Porous coated anatomic tricompartmental tibial components. The relationship between prosthetic position and micromotion. Clin Orthop Relat Res 251:189-197

3. Søballe K, Overgaard S (1996) The current status of hydroxyapatite coating of prostheses. J Bone Joint Surg Br 78:689-691

4. Stephenson PK, Freeman MAR, Revell PA, Germain J, Tuke M, Pirie CJ (1991) The effect of hydroxyapatite coating on ingrowth of bone into cavities in an implant. J Arthroplasty 6:51-58

5. Søballe K, Hansen ES, Brockstedt-Rasmussen H, Bünger C (1993) Hydroxyapatite coating converts fibrous tissue to bone around loaded implants. J Bone Joint Surg Br 75:270-278

6. Søballe K, Hansen ES, B-Rasmussen H, Jørgensen PH, Bünger C (1992) Tissue ingrowth into titanium- and hydroxyapatite-coated implants during stable and unstable mechanical conditions. J Orthop Res 10:285-299

7. Therbo M, Petersen MM, Varmarken JE, Olsen CA, Lund B (2003) Influence of pre-operative bone mineral content of the proximal tibia on revision rate after uncemented knee arthroplasty. J Bone Joint Surg Br 85:975-979
8. Petersen MM, Gehrchen M, Østgaard SE, Nielsen PK, Lund B (2005) Effect of hydroxyapatite-coated tibial components on change in bone mineral density of the proximal tibia after uncemented total knee arthroplasty. J Arthroplasty 20:516-520

9. Østgaard SE, Gottlieb L, Toksvig-Larsen S, Lebech A, Talbot A, Lund B (1997) Roentgen stereophotogrammetric analysis using computer-based image-analysis. J Biomech 30:993-995

10. Selvik G (1989) Roentgen stereophotogrammetry. A Method for the study of the kinematics of the skeletal system. Acta Orthop (Suppl) 60:1-51 (Reprint)

11. Valstar ER (2001) Digital roentgen stereophotogrammetry: development, validation and clinical application (Thesis). University of Leiden, Holland pp 1-179

12. Valstar ER, Gill R, Ryd L, Flivik G, Börlin N, Kärrholm J (2005) Guidelines for standardization of radiostereometry (RSA) of implants. Acta Orthop 76:563-572

13. Ryd L (1986) Micromotion in knee arthroplasty. A roentgen stereophotogrammetric analysis of tibial component fixation. Acta Orthop 57(Suppl): $1-80$

14. Nyström L, Söderqvist I, Wedin PÅ (1994) A note on some identification problems arising in roentgen stereophotogrammetric analysis. J Biomech 27:1291-1294

15. van der Linde MJA, Garling EH, Valstar ER, Tonino AJ, Nelissen RGHH (2006) Periapatite may not improve micromotion of knee prostheses in rheumatoid arthritis. Clin Orthop Relat Res 448:122-128

16. Ryd L, Toksvig-Larsen S (1993) Early postoperative fixation of tibial components: an in vivo roentgen stereophotogrammetric analysis. J Orthop Res 11:142-148

17. Nilsson KG, Kärrholm J, Carlsson L, Dalén T (1999) Hydroxyapatite coating versus cemented fixation of the tibial component in total knee arthroplasty. J Arthroplasty 14:9-20

18. Regnèr L, Carlsson L, Kärrholm J, Herberts P (2000) Tibial component fixation in porous-and hydroxyapatite-coated total knee arthroplasty. J Arthroplasty 15:681-689

19. Nelissen RG, Valstar ER, Rozing PM (1998) The effect of hydroxyapatite on the micromotion af total knee prostheses. A prospective, randomized, double-blind study. J Bone Joint Surg Am 80:1665-1672 